\title{
Reentrant Transitions of Ising-Heisenberg Ferromagnet on a Triangular Lattice with Diamond-Like Decorations
}

\author{
M. JAŠČUR, J. StrReČKA AND L. ČAnOvÁ \\ Department of Theoretical Physics and Astrophysics, Faculty of Science \\ P.J. Šafárik University, Park Angelinum 9, 04001 Košice, Slovak Republic \\ The mixed spin-1/2 and spin-1 Ising-Heisenberg ferromagnet on the \\ decorated triangular lattice consisting of inter-connected diamonds is in- \\ vestigated within the framework of an exact decoration-iteration mapping \\ transformation. It is shown that the diamond-like decoration by a couple \\ of the Heisenberg spins gives rise to a diverse critical behaviour including \\ reentrant phase transitions with two consecutive critical points.
}

PACS numbers: 05.50.+q, 68.35.Rh

\section{Introduction}

The quantum Heisenberg model represents a long-standing theoretical challenge in the condensed matter physics especially due to insurmountable mathematical complexities associated with a non-commutability of spin operators involved in its Hamiltonian. The non-commutability between the relevant spin operators raises a zero-point motion (quantum fluctuations) and consequently, lowdimensional antiferromagnets often exhibit a variety of exotic ground states owing to quantum phase transitions driven by the quantum fluctuations. By contrast, low-dimensional ferromagnets are usually thought of as being less affected by the quantum fluctuations.

Several low-dimensional quantum Heisenberg models can exactly be solved merely on behalf of their special geometries [1, 2]. Simple geometries, which are solely composed of inter-connecting diamonds, turned out to be a very useful testing ground for elucidating quantum properties of the low-dimensional spin systems [3]. In our previous work, we have proposed the Ising-Heisenberg model on decorated planar lattices consisting of inter-connected diamonds in order to clarify a mutual interplay between the long-range magnetic order and quantum 
fluctuations [4]. The rigorous solution revealed a surprisingly rich ground-state phase diagram even for the ferromagnetic model, which consists of the classical ferromagnetic phase (CFP), the quantum ferromagnetic phase (QFP), and the disordered phase (DP). It is worthwhile to remark, moreover, that DP originates from a peculiar geometric spin frustration related to the competition between the easy-axis and easy-plane interactions. Therefore, the purpose of the present work is to shed light on how this competition influences (dis)appearance of spontaneous ordering of two long-range ordered phases CFP and QFP.

\section{Model and exact map}

First, let us briefly describe the hybrid Ising-Heisenberg model on the decorated triangular lattice constituted by inter-connected diamonds as shown in Fig. 1. It is quite obvious that the considered lattice is formed by two different spin sites

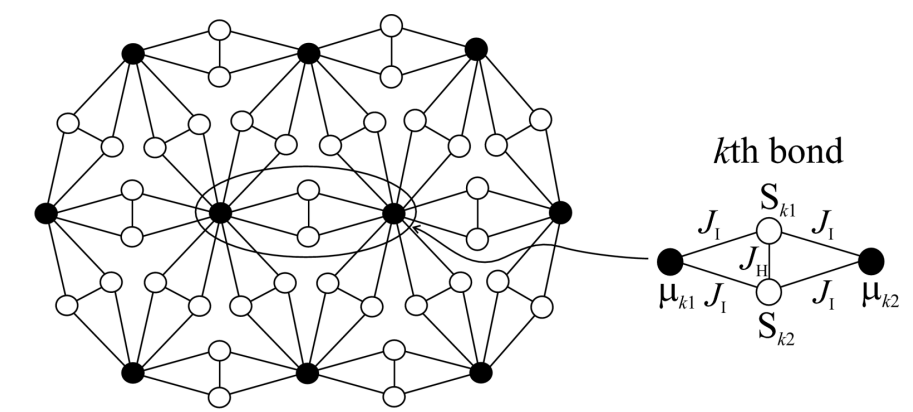

Fig. 1. The cross-section of the decorated triangular lattice consisting of interconnected diamonds. The full and empty circles illustrate lattice positions of the Ising and Heisenberg spins, respectively. The ellipse allocates an elementary cell (spin cluster) of the decorated lattice described through the bond Hamiltonian (1).

schematically depicted in Fig. 1 as full and empty circles, respectively. The former spin sites are occupied by the spin- $1 / 2$ Ising atoms described by spin operators $\hat{\mu}_{k \alpha}^{z}(\alpha=1,2)$, while the latter ones are occupied by the spin-1 Heisenberg atoms described by spin operators $\hat{S}_{k \alpha}^{\gamma}(\gamma=x, y, z)$. The total Hamiltonian can be for convenience defined as a sum of bond Hamiltonians, $\hat{\mathcal{H}}=\sum_{k} \hat{\mathcal{H}}_{k}$, where each bond Hamiltonian

$$
\begin{aligned}
\hat{\mathcal{H}}_{k} & =-J_{\mathrm{H}}\left[\Delta\left(\hat{S}_{k 1}^{x} \hat{S}_{k 2}^{x}+\hat{S}_{k 1}^{y} \hat{S}_{k 2}^{y}\right)+\hat{S}_{k 1}^{z} \hat{S}_{k 2}^{z}\right]-D\left[\left(\hat{S}_{k 1}^{z}\right)^{2}+\left(\hat{S}_{k 2}^{z}\right)^{2}\right] \\
& -J_{\mathrm{I}}\left(\hat{S}_{k 1}^{z}+\hat{S}_{k 2}^{z}\right)\left(\hat{\mu}_{k 1}^{z}+\hat{\mu}_{k 2}^{z}\right)
\end{aligned}
$$

involves all the interactions terms of one diamond-like spin cluster residing on the $k$-th bond of the triangular lattice. Above, the parameter $J_{\mathrm{H}}(\Delta)$ represents the anisotropic $X X Z$ Heisenberg interaction between the nearest-neighbouring spin-1 atoms, $\Delta$ is a spatial anisotropy in this exchange interaction, whereas the parameter $D$ measures a strength of the uniaxial single-ion anisotropy acting on 
the spin-1 atoms. Finally, the parameter $J_{\mathrm{I}}$ denotes the Ising-like exchange interaction between the nearest-neighbouring spin- $1 / 2$ and spin- 1 atoms, respectively. The afore-described Ising-Heisenberg model can accurately be treated by adopting the well-known procedure based on the exact decoration-iteration mapping transformation [4]. As a result of this mapping, the partition function $\mathcal{Z}$ of the mixed spin-1/2 and spin-1 Ising-Heisenberg model on the diamond-like decorated triangular lattice can be expressed in terms of the partition function $\mathcal{Z}_{0}$ of the spin-1/2 Ising model on the simple triangular lattice with some effective nearest-neighbour interaction $R$ :

$$
\mathcal{Z}\left(T, J_{\mathrm{H}}, \Delta, D, J_{\mathrm{I}}\right)=A^{3 N} \mathcal{Z}_{0}(T, R)
$$

Let us note that both mapping parameters $A$ and $R$ are unambiguously determined by a self-consistency condition of the mapping transformation and their explicit form is given by Eqs. 4-7 of Ref. [4]. It directly follows from the relation (2) that the hybrid Ising-Heisenberg model becomes critical if and only if its corresponding spin-1/2 Ising model on the triangular lattice becomes critical, as well. Accordingly, the exact critical temperature $T_{\mathrm{C}}$ of the hybrid Ising-Heisenberg model must obey the condition $k_{\mathrm{B}} T_{\mathrm{C}}=R / \ln 3$ ( $k_{\mathrm{B}}$ is Boltzmann's constant).

\section{Results and discussions}

The dependence of reduced critical temperature on the anisotropy parameters $\Delta$ and $D / J_{\mathrm{I}}$ is shown in Fig. 2 by keeping the ratio $J_{\mathrm{H}} / J_{\mathrm{I}}=1.0$ constant. As it can be clearly seen, the model under investigation displays a rather complex crit-
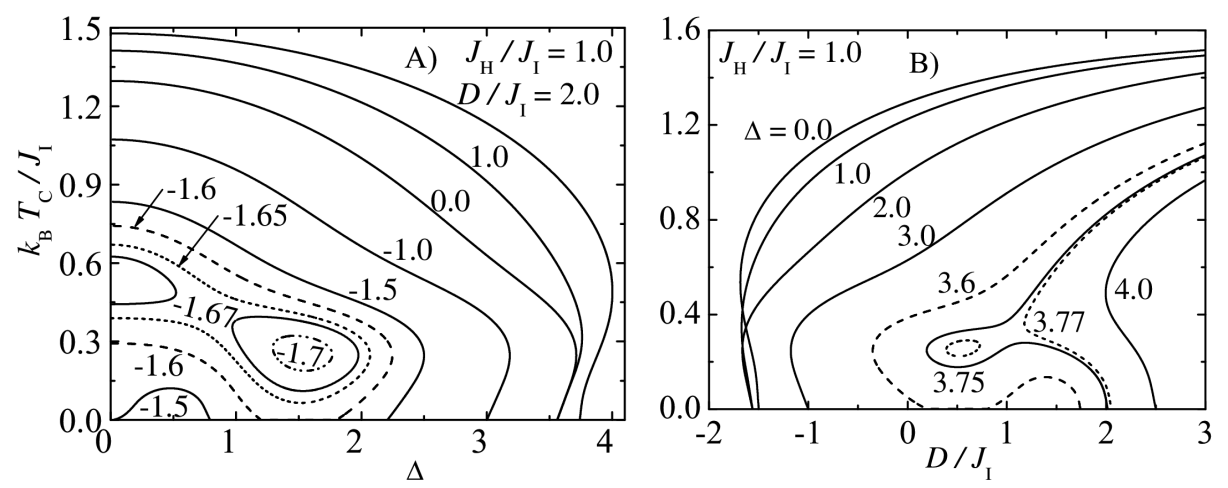

Fig. 2. (A) The critical temperature as a function of the exchange anisotropy $\Delta$ for several values of the single-ion anisotropy $D / J_{\mathrm{I}}$ and $J_{\mathrm{H}} / J_{\mathrm{I}}=1.0$. (B) The critical temperature as a function of the single-ion anisotropy $D / J_{\mathrm{I}}$ for several values of the exchange anisotropy $\Delta$ and $J_{\mathrm{H}} / J_{\mathrm{I}}=1.0$.

ical behaviour that depends very sensitively on a strength of both anisotropy parameters. Within the finite-temperature phase diagram one actually finds several regions where the system exhibits reentrant phase transitions with two consecutive 
critical temperatures, which might be even relatively well separated. Generally, the reentrance presumably occurs either if the single-ion anisotropy is of an easyaxis type $(D>0)$ and the exchange anisotropy is of an easy-plane type $(\Delta>1)$ or if the reverse is the case. This indicates that the reentrance is closely related to a competition between the easy-plane and easy-axis interactions, while the latter one is also supported by the Ising interaction $J_{\mathrm{I}}$. Besides, it is worthwhile to remark that the most interesting part of the finite-temperature phase diagram includes closed loops of phase transition lines, which separate the long-range ordered phase (either CFP or QFP) from the disordered one. Let us notice that the results displayed in Fig. 2 have been convincingly evidenced also by a detailed analysis of the spontaneous magnetization, which represents the order parameter for both ferromagnetic phases CFP and QFP. In conclusion, it should be mentioned that the critical behaviour of the Ising-Heisenberg models on different diamond-like decorated planar lattices basically depends also on a lattice topology even though all lattices have the same ground-state phase diagram regardless of their coordination number [4]. In this respect, more systematic study that would clarify this issue in detail is left for our future work.

\section{Acknowledgments}

This work was supported by the Slovak Research and Development Agency under the contract No. LPP-0107-06 and the grant VVGS PF 02/2007/F.

\section{References}

[1] E.H. Lieb, D.C. Mattis, J. Math. Phys. 3, 749 (1962).

[2] C.K. Majumdar, D.K. Ghosh, J. Math. Phys. 10, 1388 (1969); 1399 (1969).

[3] M.W. Long, S. Siak, J. Phys., Condens. Matter 2, 10321 (1990).

[4] J. Strečka, M. Jaščur, Phys. Status Solidi B 233, R12 (2002). 